1 Universidad de la Salle, Facultad de Ciencias de La Educación; Maestría en Educación de la Pontificia Universidad Javeriana. Estudiante del Doctorado Interinstitucional en Educación; correo electrónico: andreamunozbarriga@ gmail.com

\section{Diversidad cultural en la primera infancia: un reto educativo en contextos urbanos}

\section{Resumen \\ El tema de la diversidad en la educación para la primera infancia en contextos urbanos, es un reto al cual se enfrentan los maestros con la necesidad de ofrecer alternativas formativas a los grupos minoritarios, pues este es un ciclo vital de desarrollo cognitivo, social y emocional para la construcción de identidades. En tal sentido, se busca hacer un recorrido por las diversas conceptualizaciones y discursos acerca de la diversidad y su impacto en el ámbito de la educación, con el fin de ofrecer un panorama teórico que promueva la reflexión sobre la escuela como espacio de consolidación de las interacciones dialógicas entre grupos sociales.}

Palabras clave: Diversidad, educación intercultural, primera infancia, formación de maestros.

\section{Cultural diversity in early childhood: a challenge in urban education}

\section{Summary}

The topic of diversity for early childhood in urban contexts is a challenge that teachers face with the necessity of providing educational alternatives to minority groups, since this is a vital cycle of cognitive, social and emotional development and identity construction. As a consequence, this text is intended to go over the different concepts and discourses related to diversity and their impact on education with the purpose of offering a theoretical background to promote reflection towards the school as a place for strengthening interactions among social groups.

Keywords: Diversity, intercultural education, early childhood, teacher training.

\section{Diversidade cultural na primeira infância: um repto educativo em contextos urbanos}

\section{Resumo}

0 tema da diversidade na educação para a primeira infância em contextos urbanos, é um repto ao qual se enfrentam os maestros com a necessidade de oferecer alternativas formativas aos grupos minoritários, pois este é um ciclo vital de desenvolvimento cognitivo, social e emocional para a construção de identidades. Em tal sentido, procura-se fazer um percurso pelas diversas conceitualizações e discursos a respeito da diversidade e seu impacto no âmbito da educação, com o fim de oferecer um panorama teórico que promova a reflexão sobre a escola como espaço de consolidação das interacções dialógicas entre grupos sociais.

Palavras-chave: Diversidade, educação intercultural, primeira infância, formação de maestros. 


\section{Introducción}

$\mathrm{E}^{\mathrm{I}}$ fenómeno de la globalización ha generado múltiples transformaciones en las formas de comprender y significar el mundo y las relaciones sociales, promoviendo una especie de crisis generalizada de sentido en la sociedad. Una de las causas de dicha crisis tiene sus raíces en la tendencia a la homogeneización o incluso en la amenaza de la desaparición de las identidades locales, regionales o nacionales desde la imposición de modelos únicos de interpretación del mundo, en contextos donde crece una gran variedad de expresiones y formas de la alteridad.

Diversos autores exponen la importancia del tema en el mundo globalizado, y cómo este fenómeno homogeneizante refleja multiplicidad de identidades propias de la modernidad, en cuya dinámica homogeneizante se expanden y florecen viejas y nuevas construcciones de sentido, algunas de ellas resistentes a su lógica hegemónica (Rojas, 2000, p. 11).

En consecuencia, el ideal de comunidad y nación son producto del reconocimiento social, político o jurídico de diferentes formas de diversidad que han sido tradicionalmente discriminadas y excluidas. Al respecto, Rojas (2000, pp. 11-12), parafrasea a Guerrero $(1999$, p. 9), cuando afirma:

\footnotetext{
"La diversidad no sólo es la comprobación de la existencia de un gran número de grupos humanos social y culturalmente diversos, la diversidad es también - una respuesta política a los imaginarios construidos desde el poder que inculcan, especialmente en la actual fase de globalización y planetarización del mercado, perspectivas homogeneizantes, totalizadoras y universalizantes que buscan ser mostradas como paradigmas incuestionables, como símbolos de un nuevo sentido, y como únicos y posibles valores y caminos a seguir para garantizar nuestro futuro; en realidad de lo que se trata es que entendamos que la verdadera riqueza de nuestras sociedades y la garantía de su futuro, está en la variedad, en la pluralidad, en la multiplicidad y la diferencia -".
}

En este marco, la escuela actual debe ofrecer alternativas formativas a los diversos grupos sobre los principios de interculturalidad, especialmente en la primera infancia, como ciclo vital de desarrollo cognitivo, social y emocional en el que se forman las identidades, en tanto la escuela se constituye en el espacio donde se pueden nutrir las interacciones dialógicas entre grupos sociales desde visiones dinámicas de la cultura.

\section{Conceptualización de la diversidad cultural en educación}

Los primeros estudios que incorporan el tratamiento de la diversidad (desde 1882 hasta 1915) se originan a partir de la creación de la Asociación para el Estudio de la Vida e Historia del Negro en Chicago. Este hecho tuvo una enorme incidencia en la sociedad norteamericana y en el desarrollo de propuestas dirigidas a intervenir la práctica educativa.

En tal sentido, la diversidad cultural como objeto de estudio en educación es relativamente reciente y sólo se consolida en términos de educación multicultural (como se denomina en el mundo anglosajón) en la década de los 70, con la publicación de la Guía Curricular para la Educación Multiétnica (1976) que se convierte en el eje principal de numerosos estudios científicos. En este contexto, dos movimientos desarrollaron la producción científica en torno a la comprensión interracial.

Por un lado, The early ethnic studies, que se ocupó del estudio de la cultura afroamericana y, por otro, el grupo Intergroup education, dedicado al análisis de las relaciones, historia y cultura de los grupos étnicos (Banks, 1996). Estos movimientos impulsaron, por una parte, estudios orientados a la disminución de las diferencias raciales determinadas por las relaciones con la cultura dominante y, posteriormente, se dirigieron a la búsqueda de posibilidades de cambio del sistema educativo desde la idea de una escuela equitativa. A su vez, las investigaciones también se enfocaron en el análisis de la discriminación social en la escuela a partir de estudios sobre el impacto curricular de aspectos como género, raza, clase, discriminación, exclusión e inclusión social.

En Europa la educación intercultural (término que se utiliza en esta región) surgió a partir del fenómeno de la migración en 1966, vinculado principalmente con el estudio de la edu- 


\section{El término etnoeducación es reconocido oficialmente hasta la constitución de 1991 y, desde entonces, es acogido como respuesta a la necesidad de reconocimiento de los grupos sociales históricamente marginados"}

\begin{abstract}
pan miembros con una diversidad cultural manifiesta, y en relación a los cuales, existen distintos niveles de aceptación, comprensión y compromiso, que responden a premisas filosóficas y socio-políticas diferentes. Consideramos, por tanto, que el término de "educación multicultural" hace referencia a uno de los rasgos que definen al contexto en que esta educación se lleva a cabo, pero poco o nada dice de los rasgos y condiciones que definen a dicho proceso [...] La interculturalidad, sin embargo, representa una clara demarcación de la mera multiculturalidad, por cuanto penetra en la esencia misma del proceso educativo en una determinada dirección. Como superación del multiculturalismo se está potenciando en todos nuestros ámbitos escolares y sociales, el concepto de interculturalidad (interculturalismo), para explicar que es necesario ir más allá de la aceptación evidente de la existencia de diferentes culturas, buscando sobre todo el intercambio, la reciprocidad, la interacción, la relación mutua y la solidaridad entre diferentes modos de entender la vida, los valores, la historia, las conductas sociales, etc., en condiciones de influencia paritaria".
\end{abstract}

Así, a diferencia de la multiculturalidad, entendida en términos de relativismo cultural, en el que las culturas se conciben separadas porque se refieren de forma descriptiva a la existencia de grupos culturales divididos y opuestos que conviven en un mismo espacio sin una interrelación equitativa; la interculturalidad indica la existencia de relaciones complejas que involucran el desarrollo de interacciones, que más allá de incluir el reconocimiento del "otro", impulsen procesos de intercambio social, político, educativo y comunicativo mediados por prácticas y acciones particulares.

Ahora bien, los avances en el estudio de la diversidad cultural en educación y la transformación discursiva de su conceptualización a nivel global, incidieron en el contexto colombiano desde las décadas de los 70 y 80 con la llegada de la etnoeducación. Este concepto surge de la necesidad de cons- truir una sociedad más inclusiva, abierta a la diversidad y a sus variados sentidos en una cultura pluralista, por cuanto es el resultado de un proceso precedido por un largo período de evangelización de las poblaciones indígenas y por una serie de confrontaciones políticas y culturales entre el Estado y los grupos étnicos desde el siglo XVI hasta finales del siglo XX.

La historia de la educación para la diversidad se originó en el marco del movimiento social indígena, abarcando posteriormente a las comunidades afrocolombianas. El término etnoeducación es reconocido oficialmente hasta la constitución de 1991 y, desde entonces, es acogido como respuesta a la necesidad de reconocimiento de los grupos sociales históricamente marginados desde los imaginarios construidos por el poder dominante, que tradicionalmente ha legitimado un sentido único y una única forma de percibir, comprender y relacionarse con el mundo.

"La etnoeducación se inserta en un proceso social complejo en el que se manifiesta el conflicto entre las viejas formas de interpretar la realidad y aquellas que pugnan por construir nuevos imaginarios, nuevas formas de entender su condición de pluralidad que den cabida a los diferentes actores sociales que luchan por su reconocimiento y por la construcción de un nuevo sentido para la sociedad" (Rojas, 2000, p. 7).

Esta lucha por el reconocimiento y por el sentido de la identidad de un colectivo que caracteriza a la diversidad, es el espacio en el cual surge la etnoeducación.

"La etnoeducación surge como respuesta al modelo descontextualizado que caracterizó a la educación oficial, en manos de la Iglesia y del Estado, impartida en zonas indígenas a lo largo de la historia y que ha tenido como consecuencia fuertes procesos de aculturación entre la población de la mayoría de los más de ochenta pueblos indígenas que habitan el país" (Rojas, 2000, p. 5). 

nominación de la alteridad"

Aunque existen diversas conceptualizaciones, ésta se concibe principalmente como un espacio de construcción y reconstrucción de saberes desde una perspectiva intercultural, en la que se comparten voces que contribuyen a la auto-identificación, recreación y reflexión sobre las diversas formas de conocer e interpretar el mundo (Triviño, 1998, citado en Rojas, 2000).

A pesar de los avances en el estudio de la diversidad en contextos educativos y sus transformaciones discursivas, en Colombia la idea de la diferencia ha estado ligada históricamente al concepto de raza como resultado de la colonización. Un concepto que da forma a jerarquías sociales que han permitido "naturalizar" un orden social de dominación en la dinámica constitutiva del proceso de la modernidad (Quijano, 2000, citado en Ytarte, 2002). Este hecho ha marcado las representaciones acerca de las minorías como poblaciones subordinadas con características culturales particulares y diferentes de las que hacen parte de los sectores dominantes. La multiculturalidad aquí aparece como una noción ligada a la dimensión étnica.

\begin{abstract}
"La expresión actual de la multiculturalidad se halla restringida o marcada por lo étnico (multiculturalidad etnizada), a la vez que lo étnico corresponde a una concepción definida por la pervivencia de tradiciones y costumbres que se sostienen imperturbadas e imperturbables en el tiempo en el que lo indígena se constituye como paradigma (etnicidad indigenizada)" (Rojas y Castillo, 2007).
\end{abstract}

El discurso de la interculturalidad en Colombia, empieza a constituirse limitadamente sólo hasta la década de los 90 con la influencia de los estudios en el campo educativo en América Latina, y se ha conceptualizado principalmente como un proceso social representado en la expresión de la diversidad, como interacción dialógica entre todos los grupos sociales cuya lucha es el reconocimiento.
La noción ha promovido el desarrollo de proyectos educativos como la educación bilingüe intercultural (EBI), educación intercultural bilingüe (EIB), etnoeducación, educación propia, Cátedra de estudios afrocolombianos, entre otros. Estos proyectos suponen una representación de lo intercultural como una relación curricular entre conocimientos de culturas indígenas y aquellos que son "desconocidos o ajenos", en los que las culturas aborígenes se encuentran en condiciones de aislamiento (Rojas y Castillo, 2007).

En términos generales, Rojas y Castillo (2007), establecen que la interculturalidad ha estado ligada a la idea de diálogo entre culturas y saberes, sin embargo, este proceso ha sido traducido en una forma de complementar los saberes universales sin cuestionar las lógicas de representación en que se inscriben las culturas y los saberes de las comunidades minoritarias, lo cual ha generado la reproducción de las políticas de representación dominantes y una interculturalidad igualmente etnizada.

\section{Representaciones de la diversidad en la escuela y la formación docente para la primera infancia}

En la formación de los maestros y maestras se observa
la tendencia a privilegiar mucho más el ser capaces de
hablar "sobre la diversidad", "sobre el otro", "acerca del
otro", pero no el de poder "conversar con el otro" y, mu-
cho menos, el de dejar que los "otros conversen entre ellos
mismos". (Skliar: 2008, p. 3 )

La problemática de la educación para la diversidad en la escuela y los modelos educativos actuales, ha estado influenciada por las constantes tensiones que se han presentado debido a las diferentes formas de nominación de la alteridad. El término "diversidad" ha sido objeto de fuertes controversias que han dado lugar a varios tipos de discursos que indiscutiblemente 
han tenido un impacto en los sistemas educativos y particularmente en el lenguaje escolar.

Las tensiones mencionadas han tenido implicaciones que se reflejan en procesos educativos violentos o excluyentes y visiones folklóricas y relativistas que invitan a pensar en nuevas formas de representación y educación para la diferencia. Tal como lo proponen Duschatzky y Skliar (2000), se han evidenciado particularmente tres versiones de la diversidad en el pensamiento moderno: a) El otro como fuente de todo mal, b) El otro como sujeto pleno de un grupo cultural y c) El otro como alguien a ser tolerado.

La alteridad vista como la fuente de todo mal se constituyó en el modo predominante de relación social durante el siglo $\mathrm{XX}$. En tanto este fue uno de los siglos más violentos y mortíferos de la historia, la alteridad se evidencia como una forma de representación de la negación de los conflictos culturales, con el fin de regular y controlar la mirada de aquellos que representan o definen quiénes y cómo son los otros.

En esta representación se establece una lógica binaria, con la intención de visibilizar o invisibilizar al otro a partir de denominaciones que implican un componente negativo, lo cual promueve formas de narración de la diversidad, influenciadas por la búsqueda de eufemismos o denominaciones no neutras que buscan desligar las marcas identitarias, depositando en los otros estereotipos sociales.

A su vez, ha coexistido una segunda concepción acerca de la diversidad, más ligada a la representación de comunidades homogéneas fundadas en patrones culturales y con identidades plenas. La diversidad aquí es una categoría ontológica, un objeto epistemológico que supone el reconocimiento de contenidos y costumbres culturales preestablecidas (Duschatzky y Skliar, 2000).

Desde esta perspectiva, se busca defender la importancia de las sociedades plurales y democráticas por medio de representaciones radicales de culturas totalizadas que terminan alimentando un discurso homogeneizante. El multiculturalismo entonces se convierte en una manera elegante de confesar la brutalidad colonial y la falta de respuesta a la violencia y la exclusión en la modernidad (Zizek, 1998, citado en Duschatzky y Skliar, 2000).
Por último, los autores mencionan una tercera forma de representación que nutre la idea de la tolerancia en el discurso posmoderno, la cual resalta la diferencia y a su vez rechaza la conducta antisocial y opresiva. Dicha representación de la diversidad no permite examinar las culturas críticamente en contextos concretos y, por tanto, impide establecer un vínculo social, que aunque de naturaleza conflictiva, implica una responsabilidad ética y política.

En lo que se refiere a la educación para la primera infancia, el estudio de la diversidad cultural en contextos urbanos es un objetivo primordial, puesto que se evidencia la necesidad de respuestas formativas que logren atender las necesidades de niñas y niños pertenecientes a grupos minoritarios. En estos contextos se encuentran niños inmigrantes y otros nacidos en la ciudad que provienen de comunidades indígenas. Estos niños enfrentan dificultades debido a las tensiones entre la cultura dominante y la minoritaria, así como presiones derivadas de actos violentos, racistas y discriminatorios en contextos excluyentes. Delgadillo et al $(2011$, pp. 11, 12) ilustran la complejidad de la problemática:

\begin{abstract}
“Cada pueblo originario entra en una dinámica de tensión entre lo propio y las prácticas culturales urbanas, que vivida en la primera infancia, implica la socialización bajo otros patrones de crianza diferentes a los de la propia ancestralidad, el cambio de lengua materna originaria por el español, una dieta diferente a la de las prácticas alimentarias tradicionales y la construcción de una identidad híbrida y subalternizada frente al origen de la propia familia. A esta circunstancia se agrega el que en la mayoría de los escenarios de formación de la infancia, los contenidos curriculares no desarrollan lineamientos diferenciales y menos interculturales, y su organización por competencias, estandariza aún más la pertinencia cultural, de enseñabilidad y la diversidad de formas de aprendizaje, convirtiéndose estos establecimientos en dispositivos de reproducción de la discriminación".
\end{abstract}

La etapa de formación de los niños y niñas de cero a seis años es un ciclo vital de desarrollo cognitivo, social y emocional, y debe involucrar procesos formativos que favorezcan el desarrollo de interacciones sociales y la articulación de sus intereses, necesidades y particularidades a la escuela. Esto implica alejar- 
se de las formas de nombrar y conceptualizar la interculturalidad que han sido tradicionales desde las culturas dominantes, y acercarse más al análisis de las representaciones de la interculturalidad desde los discursos de los grupos étnicos.

Uno de los desafíos de la educación actual para la primera infancia es la formación docente desde y para la diversidad, pues los maestros se enfrentan diariamente a la necesidad de encontrar alternativas de formación que consoliden propuestas pedagógicas, no sólo para el reconocimiento de las minorías, sino para el establecimiento de interacciones dialógicas que den sentido a la diversidad en el aula.

Los discursos y las racionalidades frente a la diferencia que tradicionalmente han coexistido en los espacios y las políticas educativas, han influido en las perspectivas alrededor de la formación y el diseño de programas y modelos pedagógicos que evidencian determinadas posiciones frente a la cultura.

En este sentido, López (2002) evidencia dicha problemática al establecer que las tradiciones técnico-positivista, interpretativo-hermenéutica y crítico-emancipatoria, han condicionado el rol de los profesores frente a la diversidad cultural.

En el marco de la tradición técnicopositivista, la realidad cultural se presenta desde una visión fragmentada en la que las culturas aparecen desconectadas entre sí y se abordan aisladamente. Aquí prevalece un modelo cultural determinado como universal que justifica la dominación de unas culturas sobre otras; la educación se asume como un proceso que garantiza la permanencia y asimilación de la cultura dominante en la escuela, como sistema social que legitima la reproducción de la superioridad de una cultura ante la otra. Desde esta perspectiva, la cultura es esencialista y estática, en la medida en que rechaza la pluralidad como fenómeno que amenaza la estabilidad y sólo promueve la tolerancia entre culturas separadas.

Bajo esta mirada, el currículum es de carácter etnocéntrico y surge como representación de la cultura hegemónica, por cuanto es diseñado a partir de saberes y valores aceptados desde los grupos dominantes. La enseñanza, por su parte, se constituye en una actividad predecible en la que se controlan las conductas para evitar el conflicto, y los roles de docentes y estudiantes se rigen por los intereses de las mayorías en un orden jerarquizado. A su vez, la formación del profesorado se basa en lo comportamental con énfasis en el desarrollo de la actuación o competencia.

Estos hechos generan formas de violencia en la escuela que promueven la marginación y la pérdida de la identidad de los niños minoritarios, mientras que los maestros hacen esfuerzos por trasladar las propuestas curriculares de carácter homogéneo a la práctica, con el fin de mantener el orden establecido.

Desde la tradición interpretativo-hermenéutica, la escuela se compromete con el cambio de significados subjetivos y con el establecimiento de relaciones más positivas entre individuos, en tanto la realidad está mediada por los significados que individuos y grupos otorgan a los hechos. Aquí se asume la escuela como el espacio que posibilita la comunicación intercultural y el respeto por la diferencia, a partir de la tolerancia y, por otra parte, la educación se consolida como un proceso de intercambios que favorece el respeto y la comunicación.

En esta misma línea, las propuestas curriculares se producen desde la práctica, porque es el lugar donde surge la cultura y se intercambian significados e interpretaciones. La cultura es dinámica y dependiente de interpretaciones personales y, por tal motivo, el rol del profesor es activo y se adapta a situaciones cambiantes en procesos de interacción en los que se adapta el currículum a las singularidades de los alumnos.

Por su parte, la formación de los docentes se configura desde un discurso humanista en el que se pretende articular la teoría y la práctica, al tiempo que busca reducir los prejuicios culturales. Aquí López (2002), resalta que dicha formación sigue estando caracterizada por lo monocultural, pues no es generalizada y se dirige a grupos particulares; agrega que desde esta tradición no se consideran factores sociales, políticos $\mathrm{y}$ económicos que intervienen en las interacciones entre los individuos, desconociendo el contexto en que éstas se producen y su influencia en la configuración de las interpretaciones. En este sentido, la diversidad no puede centrarse únicamente en las construcciones subjetivas sin modificar las condiciones reales de la vida social.

Por último, en la tradición socio-crítica se asume que las estructuras dominantes y los sistemas de poder reproducen las 


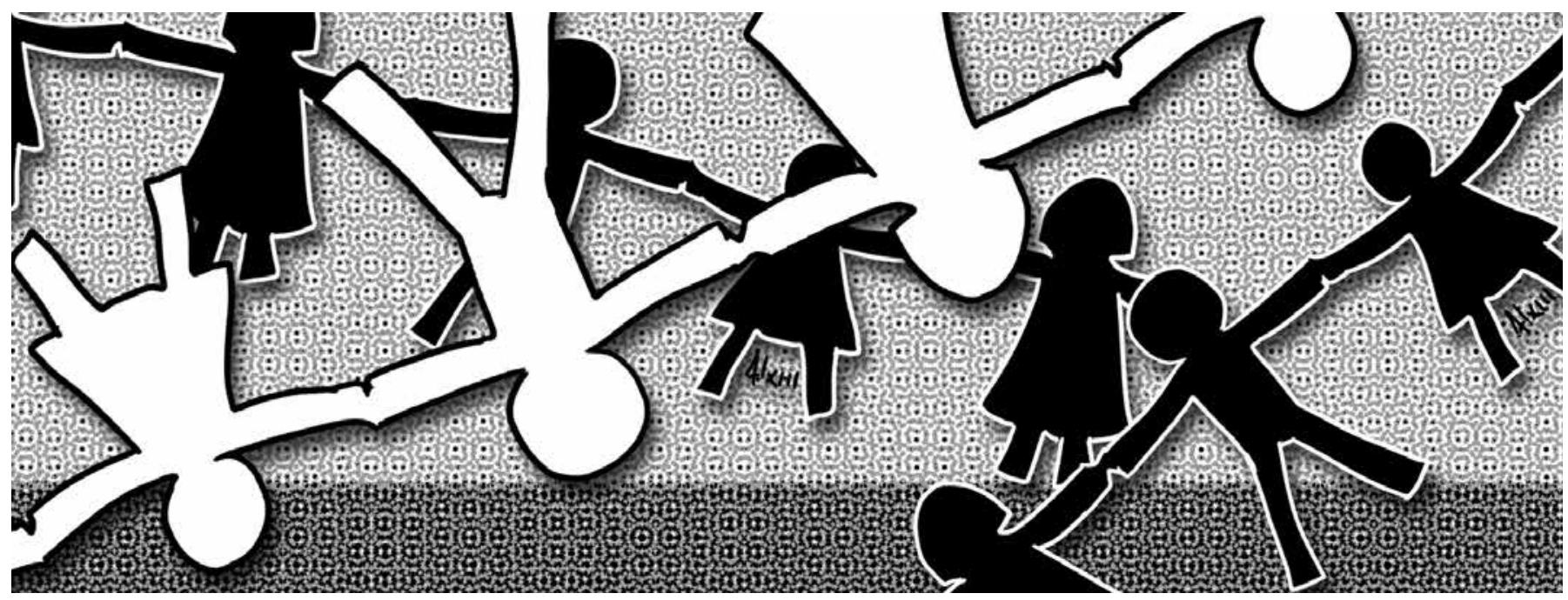

situaciones de desigualdad de los grupos culturales y, sobre esa base, la cultura debe ser abordada en articulación con los sistemas con los que interactúa. Este es un ideal por alcanzar en los contextos educativos, ya que la escuela está llamada a adoptar modelos menos violentos que realmente produzcan un cambio a nivel político, económico y social. En este punto, propone integrar la diversidad cultural a los currículos por medio de procesos de deliberación y corresponsabilidad comunitaria como procesos en los que el docente tiene un rol vital como transformador social. A este respecto López (2002, p. 97) afirma que:

\footnotetext{
"Educar para la diversidad cultural desde la perspectiva crítica, es educar para el cambio social y estructural que nos conducen a tomar conciencia de las interacciones que mantenemos con los demás y las condiciones en que éstas se producen. Este conocimiento impulsa la reciprocidad y favorece los intercambios, haciendo posible los acuerdos políticos y axiológicos necesarios para garantizar mayores cotas de libertad, igualdad y justicia social. Pero estos acuerdos requieren de una reflexión crítica que para Bolívar (1992) es ante todo una reflexión discursiva que incorpora criterios ético-morales y que permite analizar la práctica y fines educativos, las actividades que pueden contribuir a satisfacer las necesidades y propósitos humanos de justicia-equidad".
}

De esta manera, la formación de los profesores está basada en el desarrollo de procesos de indagación crítica y cooperativa del fenómeno multicultural. Sin embargo, en este modelo no hay cabida para la postura intercultural, pues aquí se resaltan más las diferencias que las compatibilidades y se promueve un concepto de cultura que continúa siendo cerrado y estático, puesto que se deja de lado la diversidad interna de cada grupo y la interrelación entre culturas.

Además de las posturas descritas, López (2002) menciona una cuarta tendencia que parece estar surgiendo actualmente, la cual involucra una visión más compleja y sistémico-global de la cultura. Ésta se centra en el humanismo como enfoque ligado a factores cognitivos y sociopolíticos, en donde lo multicultural está ligado a la parte más interna del ser humano.

La formación de maestros se caracteriza, bajo esta perspectiva, por la maduración del docente como persona y como experto en relaciones interpersonales, en el marco de una comunicación didáctica:

\footnotetext{
"En este caso, estaríamos planteando la posibilidad de situar la búsqueda de nuevas formas para el entendimiento mutuo en escenarios que van más allá del marco cultural y que se nos sitúan en esa identidad profunda, no aprendida, común y transcultural, que está mas allá del yo socializado" (López, 2002, p. 100).
} 
Es posible afirmar que en Colombia, especialmente en las zonas urbanas, se evidencia la necesidad de una formación docente más profunda sobre el sentido y el valor práctico de la diversidad"

En este marco, la educación se asume como en un proceso en el que lo personal y lo socio-local se proyecta en lo global y viceversa, donde la escuela se constituye en el espacio para la transformación social y la justicia social desde el establecimiento de acuerdos y el entendimiento mutuo en procesos dialógicos, críticos y de auto-observación:

\begin{abstract}
"La interculturalidad se consigue no sólo cambiando las estructuras, sino a los individuos, compatibilizando su conciencia crítica y su capacidad de autoobservación para acceder a esa identidad más profunda que le aproxima al resto de la humanidad con su participación social y comunitaria" (López, 2002, p. 102).
\end{abstract}

Tomando dichas tendencias como base, es posible afirmar que en Colombia, especialmente en las zonas urbanas, se evidencia la necesidad de una formación docente más profunda sobre el sentido y el valor práctico de la diversidad, para enfrentar el fracaso y los conflictos socio-afectivos de los niños y niñas de grupos minoritarios.

\section{El lenguaje como alternativa de formación desde y para la diversidad}

"Las palabras significan lo que los seres humanos acuerdan conjuntamente que signifiquen, se pueden crear nuevas palabras cuando hagan falta y se pueden combinar para expresar una variedad infinita de significados. El lenguaje nos permite compartir pensamientos sobre nuevas experiencias y organizar la vida en común como ninguna otra especie puede hacer. Esta función del lenguaje permite concebirlo desde

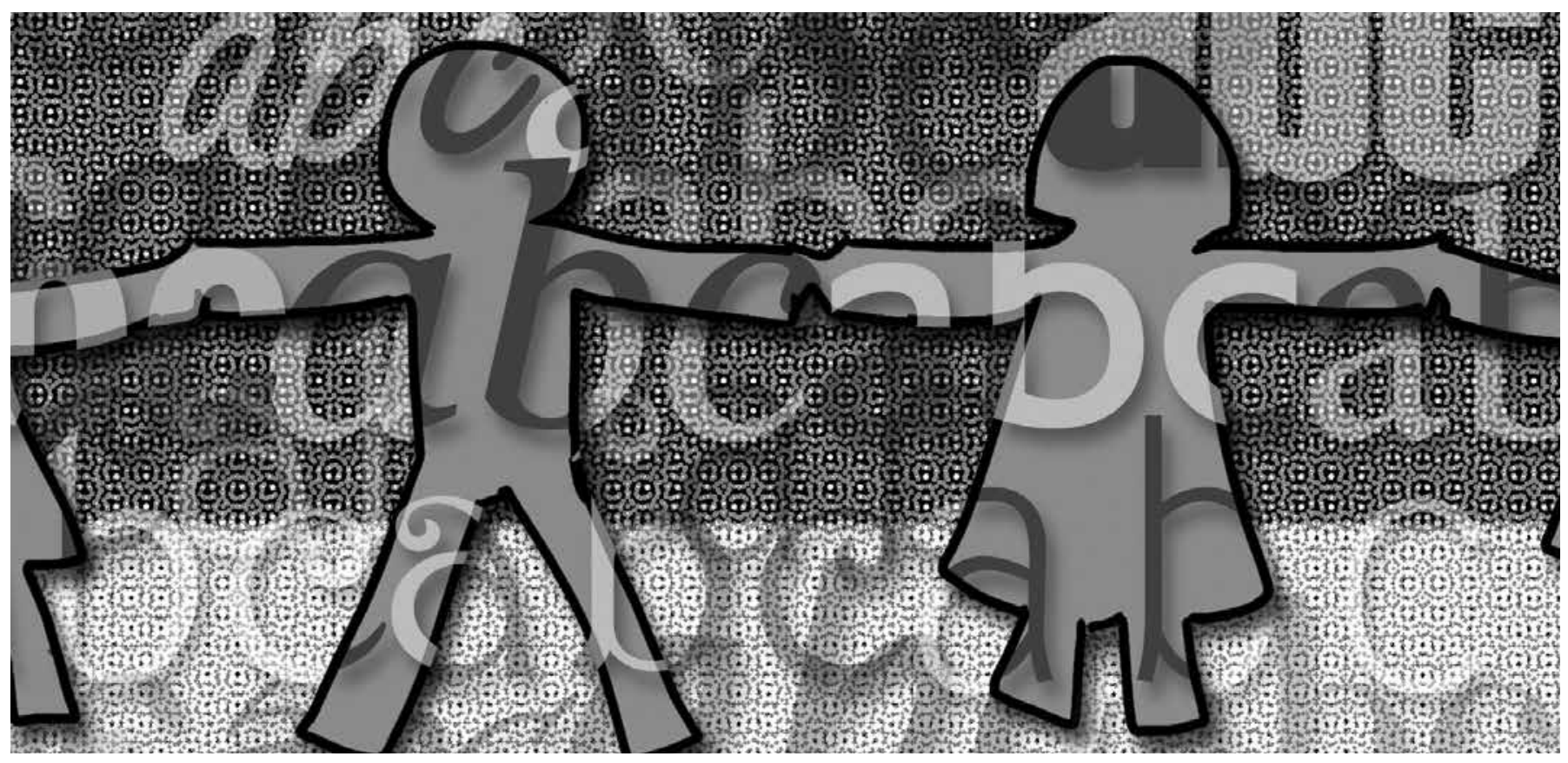


una perspectiva distinta a la instrumental (transmisión de información entre personas) a una perspectiva de interacción dialógica [...] el lenguaje está diseñado para hacer algo mucho más interesante que transmitir información con precisión de un cerebro a otro: permite que los recursos mentales de varios individuos se combinen en una inteligencia colectiva y comunicadora que permite a los interesados comprender mejor el mundo e idear maneras prácticas de tratar con él [...] empleamos el lenguaje para convertir el pensamiento individual en pensamientos y acciones colectivas". (Mercer, 2000, p. 20).

El lenguaje es una de las características fundamentales del género humano y tiene un papel fundamental en la apropiación de la cultura y la incorporación de los individuos en la sociedad; por tanto, se constituye en un elemento esencial de los procesos formativos en contextos de diversidad cultural. A través del lenguaje las niñas y niños potencian habilidades cognitivas y sociales que les permiten construir sus propias identidades, en cuanto éstas se desarrollan en las relaciones dialógicas que se establecen en la escuela.

La relación identidad y reconocimiento desde el lenguaje aquí se asume a partir de la posición de Taylor (1993, pp. 18-19):

\begin{abstract}
"La identidad se crea dialógicamente, en respuesta a nuestras relaciones, e incluye nuestros diálogos reales con los demás. Por tanto, es falsa la dicotomía que plantean algunos teóricos de la política, entre los individuos atomistas y los individuos socialmente conformados. Si la identidad humana se crea y se constituye dialógicamente, entonces el reconocimiento público de nuestra identidad requiere una política que nos dé margen para deliberar públicamente acerca de aquellos aspectos de nuestra identidad que compartimos o que potencialmente podemos compartir con otros ciudadanos. Una sociedad que reconozca la identidad individual será una sociedad deliberadora y democrática, porque la identidad individual se constituye parcialmente por el diálogo colectivo".
\end{abstract}

Para Taylor (1993), la identidad se moldea por el reconocimiento como necesidad humana vital que surge de las diferentes formas de expresión de los lenguajes humanos.

\begin{abstract}
"El que yo descubra mi propia identidad no significa que yo la haya elaborado en el aislamiento, sino que la he negociado por medio del diálogo, en parte abierto, en parte interno, con los demás. Por ello, el desarrollo de un ideal de identidad que se genera internamente, atribuye una nueva importancia al reconocimiento. Mi propia identidad depende, en forma crucial, de mis relaciones dialógicas con los demás" (Taylor, 1993, p. 55).
\end{abstract}

El lenguaje como facultad humana permite entonces representar la realidad a partir de una diversidad de símbolos y signos lingüísticos; a su vez, implica el desarrollo de procesos ligados al pensamiento y la acción como elementos determinantes en la enseñanza y el aprendizaje.

El concepto de facultad se entiende en referencia a la acción, dado que ésta evidencia la capacidad que se tiene para un conocimiento en un contexto dado, siendo éste un espacio vital que permite propiciar o restringir el ejercicio de las capacidades humanas. Dicho de mejor manera:

"Todo conocimiento implica una ardua elaboración de conceptos, relaciones y explicaciones. Estos procesos son el resultado pertinente del despliegue de la naturaleza humana, entendida como la potencia pasiva que direcciona las construcciones cognitivas, las jerarquías en los niveles de conocimiento y las complejidades en las diversas formas de actuación" (Torres, 2002, p. 30).

El lenguaje a su vez está ligado al pensamiento desde el concepto de competencia, en el que el lenguaje se constituye como una propiedad humana con origen en la razón (el lenguaje como expresión del pensamiento):

"Sin lenguaje simplemente no habría concepto alguno ni, por tanto, objeto alguno: no existe propiamente un mundo ya dado con independencia del conocimiento y del lenguaje [...] la lingüisticidad atraviesa, por tanto, al ser humano y al mundo, y en este sentido el lenguaje no sólo separa y contrapone, sino que también los une o mejor, los reúne: y así del mundo que se refleja en el hombre, nace entre ambos la lengua que vincula al hombre con el mundo y que fecunda a éste por aquel. Hombre y mundo se dan, pues, en el interior de un diálogo que los constituye" (Garagalza, 2003, p. 6). 
Así entonces, es en el lenguaje donde los niños construyen su realidad y se relacionan con el mundo:

"La competencia es un efecto de la experiencia social, de las necesidades y de las motivaciones; e integra actitudes, valores y motivaciones relacionadas con la lengua, con sus características y usos, con su interrelación con otros códigos. De manera que, si existe la realidad y el lenguaje se refiere a ella, también existe la comunicación y el lenguaje pasa a través de ella" (Bustamante, 2003, p. 122).

En esta medida, el lenguaje tiene una doble perspectiva: individual y social. A partir del lenguaje los niños se constituyen como seres individuales con características concretas, y en sus interacciones conocen y construyen la realidad. Esta particularidad del lenguaje lo constituye en una práctica sociocultural vital para la formación desde y para la diversidad, porque es allí donde se construyen los significados acerca de la cultura:

"En cuanto a su valor social, el lenguaje se torna, a través de sus diversas manifestaciones, en eje y sustento de las relaciones sociales. Gracias a la lengua y la escritura, por ejemplo, los individuos interactúan y entran en relación unos con otros con el fin de intercambiar significados, establecer acuerdos, sustentar puntos de vista, dirimir diferencias, relatar acontecimientos, describir objetos. En fin, estas dos manifestaciones del lenguaje se constituyen en instrumentos por medio de los cuales los individuos acceden a todos los ámbitos de la vida social y cultural" (MEN, 2003, p. 19).

Siguiendo a Halliday (1982), el lenguaje es un hecho social y surge en la vida del individuo mediante un intercambio continuo de significados y significantes. Partiendo de esa base, la cultura es una construcción semiótica que: "se transmite al niño primero y antes que nada mediante el lenguaje, en el curso de la interacción cotidiana en las agencias socializadoras claves de la familia, el grupo de iguales y la escuela" (Halliday, 1982, p. 163).

Por otra parte, en el contexto de la diversidad en educación, el lenguaje, asumido como práctica sociocultural, a su vez brinda la posibilidad de estudiar los lugares en los que se producen y reproducen las jerarquías sociales y sus funciones en el contexto, la sociedad y la cultura (Van Dijk, 2004). De esta manera, toda reflexión sobre la diversidad ha de contemplar las formas en que se nombra y se caracteriza la diferencia, puesto que en esas construcciones de sentidos y significados determinamos su valor social y establecemos determinadas interacciones.

Vale la pena entonces pensar también en el estudio social del discurso como una alternativa para establecer diferencias epistémicas entre comunidades y las representaciones que éstas tienen de la cultura y su incidencia en la constitución de la realidad educativa.

"Esa es la razón de que nos hayamos centrado en la palabra. Y en cómo la palabra, en tanto que modela un lenguaje sobre los "otros", tiene como efecto la construcción de una práctica educativa específica sobre ellos. De algún modo, la pedagogía actualiza los elementos culturales de su "época" y los transforma en contenidos educativos. Aunque no inventa la cultura, la moldea para hacerla comprensible a aquellos que deben aprenderla. En efecto, el lenguaje construye y estructura una determinada forma de entender lo que la cultura y la diversidad significan, y da contenido a las formas de sociedad que ella representa" (Ytarte, 2002, p. 253). 


\section{Referencias}

Bustamante, G. (2003). Las competencias en la educación colombiana. El concepto de competencia III. Un caso de recontextualización. Bogotá: Alejandría.

Delgadillo, I. et al. (2011). Tendiendo puentes entre culturas. Recuperación de una experiencia de formación de maestros y maestras de jardines infantiles indigenas de Bogotá. Bogotá: Secretaría Distrital de Integración Social.

Duschatzky, S. y Skliar, C. (2000). La diversidad bajo sospecha. Reflexiones sobre los discursos de la diversidad y sus implicaciones educativas. Cuadernos de Pedagogía de Rosario, año IV, No. 7.

Garagalza, L. (2003). Filosofía y lenguaje en la obra de Wilhelm von Humboldt. Revista Internacional de los Estudios Vascos, 48(1). Obtenido desde www.euskomedia.org/PDFAnlt/riev/48237248. pdf.

Halliday, M. (1982). Interpretación social del lenguaje y del significado. Méjico: Fondo de Cultura Económica.

López, M. (2002). Diversidad sociocultural y formación de profesores. Bilbao: Ediciones Mensajero, S.A.U.

MEN. (2003). Estándares básicos de competencias del lenguaje. Formar en lenguaje: apertura de caminos para la interlocución. Obtenido desde http://www.eduteka.org/pdfdir/MENEstandaresLenguaje2003.pdf
Mercer, N. (2000). Palabras y mentes. Cómo usamos el lenguaje para pensar juntos. Barcelona: Paidós.

Rojas, A. (2000). La etnoeducación en la construcción de sentidos sociales. Universidad del Cauca. Colombia: Imprenta patriótica del Instituto Caro y Cuervo.

Rojas, A. y Castillo, E. (2007, Mayo-Agosto). Multiculturalismo y políticas educativas en Colombia, ¿interculturalizar la educación? Revista Educación y pedagogía, Vol. XIX. No. 48.

Skliar, C. (2008). ¿Qué pretendemos con la idea de "diversidad”? Construyendo una escuela inclusiva. Encuentro inspectorial de equipos de gestión y conducción escolar. Obtenido desde www. salesianoslitoral.org.ar/files/sei/2008

Taylor, C. (1993). Multiculturalismo y la politica de reconocimiento. México: Fondo de Cultura Económica.

Torres, E. (2001). El concepto de competencia. Una mirada interdisciplinar. Tomo I, pp. 24, 25. Bogotá: Alejandría.

Van Dijk, T. (2004). Discurso y dominación. Grandes conferencias en la Facultad de Ciencias Humanas. Bogotá: Universidad Nacional.

Ytarte, R. (2002). Análisis de la educación intercultural y propuesta de un modelo de pedagogía intercultural. Tesis doctoral. Universidad de Barcelona. Obtenido desde http://ldei.ugr.es/cddi/uploads/ tesis/Ytarte2002.pdf 


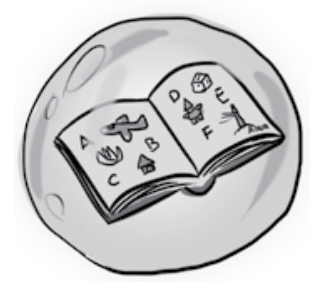

